\title{
Stenosi dell'arteria renale: rivascolarizzazione percutanea o terapia medica?
}

\author{
Maria Capuano ${ }^{1}$, Giuseppe Morelli Coppola ${ }^{2}$, Marco Terribile², Francesco Arienzo², \\ Marco Morelli Coppola ${ }^{2}$, Roberta Rossano ${ }^{3}$, Luca Apicella ${ }^{3}$
}

${ }^{1}$ Unità Operativa Nefrologia e Dialisi, Ospedale dei Pellegrini, Napoli

${ }^{2}$ Radiologia Vascolare, Ospedale dei Pellegrini, Napoli

${ }^{3}$ Cattedra di Nefrologia Università Federico II, Napoli

\begin{abstract}
RENAL ARTERY STENOSIS: PERCUTANEOUS REVASCULARIZATION OR MEDICAL THERAPY?
Abstract. Renal artery stenosis (RAS) is caused by atherosclerosis in $\mathbf{9 0 \%}$ of the cases while it is related to fibromuscolar dysplasia in almost $10 \%$ of the cases. RAS may cause malignant hypertension and reduction of kidney function. Therefore it is important to carry out an optimal therapy to reduce complications. There is general agreement on the efficacy of a strict medical therapy. On the contrary, the usefulness of a contemporary percutaneous revascularization intervention to ameliorate BP and GFR is questionable. Thus we searched the literature using PubMed by entering the search terms "renal artery stenosis, atherosclerotic renal artery stenosis, renovascular hypertension". In this review we analyze the epidemiology, physiopathology, diagnosis and, in particular, the different treatment options on the basis of the more recent clinical trials.
\end{abstract}

Key words: Renal artery stenosis, Atherosclerotic renal artery stenosis, Renovascular hypertension, Percutaneous revascularization

Conflict of interest: None.

Ricevuto: 1 Gennaio 2013; Accettato: 30 Gennaio 2013

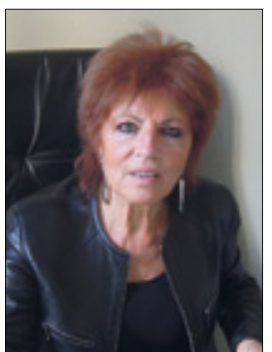

\section{Introduzione}

La stenosi dell'arteria renale (RAS) può essere causa di ipertensione maligna nonché di riduzione della funzionalità renale. È pertanto di primaria importanza mettere in atto una terapia ottimale. Non vi è un generale consenso sull'efficacia della sola terapia medica e/o rivascolarizzazione percutanea mediante Maria Capuano radiologia interventistica. La radiologia interventistica della RAS include l'angioplastica percutanea, lo stenting dell'arteria renale e lo stenting medicato dell'arteria renale. È comunque ancora molto dibattuto se sia preferibile effettuare la rivascolarizzazione percutanea rispetto alla sola tradizionale terapia medica.

Abbiamo pertanto effettuato una ricerca della letteratura utilizzando PubMed e selezionando i termini "renal artery stenosis, atherosclerotic renal artery stenosis, renovascular hypertension. Sono stati conseguentemente analizzate 14 pubblicazioni scientifiche selezionate in quanto inerenti la terapia della RAS.

\section{Epidemiologia}

L'ipertensione renovascolare è responsabile di circa il $2 \%$ dei casi di ipertensione arteriosa. Nel $90 \%$ dei casi è da correlarsi alla RAS su base arteriosclerotica (1). La prevalenza di RAS aterosclerotico aumenta con l'età e coinvolge solitamente l'ostio ed il terzo prossimale dell'arteria renale. L'ipertensione renovascolare su base aterosclerotica interessa circa il 7\% degli adulti di età $>65$ anni (2). In quasi il $10 \%$ dei casi la RAS è causata da displasia fibromuscolare, più frequente nel sesso femminile e con interessamento precipuo del tratto distale.

Più rare cause di RAS sono la vasculite, il tromboembolismo e la neurofibromatosi.

\section{Fisiopatologia}

In Figura 1 è rappresentato il meccanismo patogenetico dell'ipertensione da RAS ed i conseguenti danni d'organo. Tale meccanismo patogenetico, come descritto da Goldblatt nel 1930 è perfettamente coerente con l'ipertensione renovascolare da displasia fibromuscolare. Non è invece congruente con la fisiopatologia dell'ipertensione su base aterosclerotica i cui meccanismi sono molteplici e più complessi. La cascata di eventi origina, come ampiamente dimostrato dagli esperimenti animali, dalla diminuita perfusione renale con la conseguente produzione di renina.

Il ruolo causale più rilevante è svolto dall'angiotensina II con effetti non solo ipertensivanti ma anche proinfiammatori e tossici sul sistema cardiovascolare (3). 


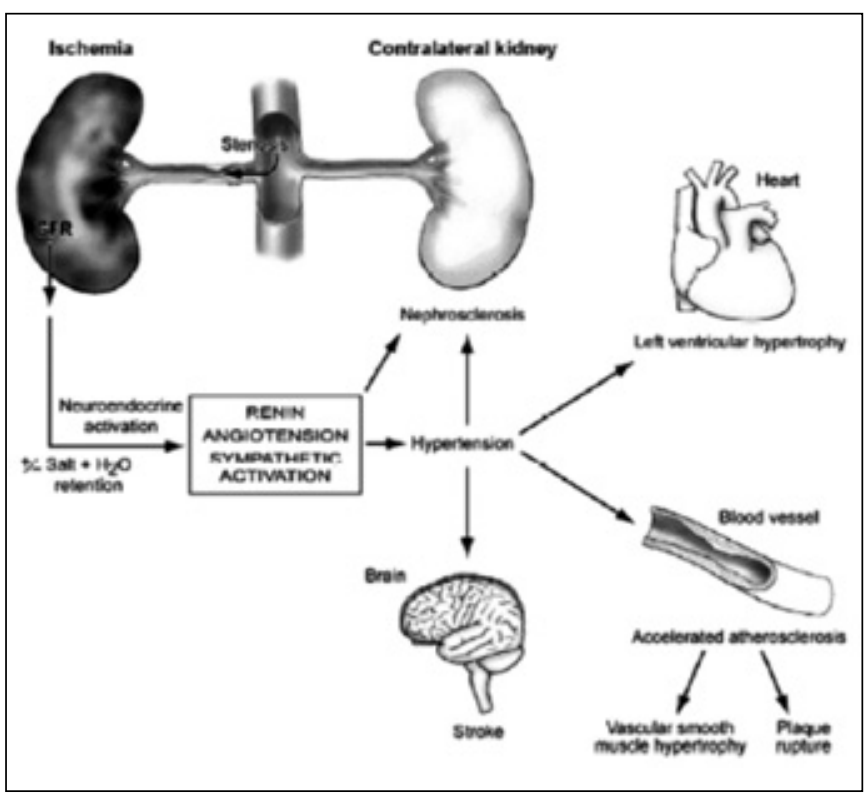

Fig. 1 - RAS, Sistema renina-angiotensina-aldosterone e danni d'organo.

\section{Diagnosi}

Sono svariate le metodiche di screening per la RAS ed includono la angioRMN, la angioTAC, l'ecocolor Doppler, la scintigrafia renale con captopril, il dosaggio della reninemia, il dosaggio della renina dalla vena renale e l'angiografia. L'angiografia rimane il gold standard, in particolare nei casi in cui gli altri accertamenti diano risultati dubbi. A causa delle basse specificità e sensibilità non sono più comunemente utilizzate la scintigrafia renale con captopril, il dosaggio della reninemia e il dosaggio della renina dalla vena renale. L'ecocolor Doppler può raggiungere una sensibilità del $97 \%$ ed una specificità del $98 \%$ (4-6). Vi possono essere però problemi tecnici legati all'obesità, alla posizione del rene e alla presenza di gas intestinale. Sia l'angioRMN che l'angioTAC hanno una sensibilità e specificità $>90 \%$ (7-9). L'angioRMN rispetto all'angioTAC ha il vantaggio di non necessitare di mezzo di contrasto iodato e dell'assenza di esposizione a radiazioni. Vi è però da considerare che l'uso di gadolinio nella RMN può comportare il rischio di fibrosi nefrogenica sistemica nei pazienti con GFR $<30 \mathrm{~mL} / \mathrm{min}$ (10). È stata recentemente proposta (11) nella patologia renovascolare aterosclerotica, come marker predittivo dell'efficacia della rivascolarizzazione percutanea, l'esecuzione della BOLD RMN che ha il vantaggio di non prevedere l'utilizzo del gadolinio. Infine la valutazione del gradiente pressorio a livello della stenosi, effettuato mediante cateterismo delle arterie renali, pur non essendo di semplice attuazione, può consentire di identificare le stenosi emodinamicamente significative e quindi suscettibili di efficace trattamento di rivascolarizzazione (12).

\section{Terapia}

Molto dibattuta è la problematica se sia preferibile nella RAS con stenosi severa $(>70 \%)$ la sola terapia medica oppure la terapia medica con rivascolarizzazione percutanea. La sola angioplastica percutanea è scarsamente utilizzata per l'alta frequenza di ristenosi. Lo stenting ha notevolmente migliorato il decorso clinico rispetto alla semplice angioplastica (13) con sempre minori effetti collaterali. Lo stenting medicato dell'arteria renale non ha ancora dimostrato la sua superiorità in riferimento al rischio di ristenosi.

È comunque difficile prevedere il decorso clinico dopo rivascolarizzazione percutanea e quantificare l'efficacia di un trattamento di radiologia interventistica addizionale. L'efficacia del trattamento è stata valutata considerando come target il miglioramento della PA, il miglioramento del GFR, la riduzione della terapia antipertensiva.

Secondo gli studi di Webster et al (14) non vi è evidenza scientifica che dimostri un beneficio clinico né per l'angioplastica né per lo stenting.

Van Jaarsveld et al (15) hanno effettuato uno studio su 56 pazienti con RAS che permanevano ipertesi malgrado l'utilizzo di almeno 2 farmaci antipertensivi. Essi hanno riscontrato un miglioramento dei valori pressori nei pazienti ipertesi sottoposti ad angioplastica rispetto ai controlli sottoposti alla sola terapia medica. Tale miglioramento è però scomparso dopo 3 mesi di osservazione.

Yusuf et al (16) hanno rilevato che un'ottimale gestione medica dell'ipertensione renovascolare è comunque in grado di ridurre le complicanze.

Cianci et al (17) hanno effettuato uno studio osservazionale su 93 pazienti; 53 di essi sono stati sottoposti a stenting dell'arteria renale più terapia medica mentre i restanti 40 alla sola terapia medica. È stato rilevato un migliore outcome renale, controllo pressorio e riduzione della frequenza di restenosi quando si utilizzava nel primo gruppo la terapia con ace-inibitori e sartanici e nel secondo gruppo quando si utilizzavano $\mathrm{i}$ sartanici e i betabloccanti.

D'altronde è stato recentemente evidenziato (18) che l'utilizzo di farmaci inibitori del sistema renina-angiotensina si dimostra ugualmente efficace e privo di effetti collaterali di rilievo anche nei pazienti con stenosi bilaterali e severe delle arterie renali.

Carr et al (19) riportano una stabilizzazione o miglioramento della funzione renale e della pressione arteriosa con la rivascolarizzazione, quando correttamente effettuata.

Arthurs et al (20) rilevano positivi risultati con la radiologia vascolare interventistica, riscontrando un significativo rallentamento del declino del GFR. Riscontrano anche un temporaneo miglioramento dei valori pressori nei primi 6 mesi.

Rabbia et al (21), invece, in uno studio di meta-analisi, non riscontrano alcun maggiore beneficio nel trattamento di rivascolarizzazione rispetto alla sola terapia medica, utilizzando come target del trattamento la stabilizzazione della funzione renale ed il controllo pressorio.

Steichen et al (22) in una review pubblicata nel 2010 rilevano che nella maggior parte dei casi sia preferibile la sola terapia medica. Consigliano invece la rivascolarizzazione quando vi sia ipertensione resistente alla terapia medica o peggioramen- 
to della funzione renale.

Jaff et al (23) in un recente studio su 202 pazienti (Hercules Trial) sottoposti a stenting dell'arteria renale, rilevano una significativa riduzione della PA sistolica a 9 mesi dall'intervento. Gli studi di maggiore rilievo sono però quelli riportati nella Tabella I.

Sono i dati di 4 grossi trial controllati e randomizzati messi in atto allo scopo di chiarire se sia preferibile la sola terapia medica o la terapia medica con la rivascolarizzazione percutanea.

Lo studio EMMA (24) è uno studio multicentrico su 49 pazienti con RAS $>60 \%$, ipertensione arteriosa e GFR $>50 \mathrm{~mL} /$ min divisi in 2 gruppi (gruppo terapia medica vs gruppo terapia medica + rivascolarizzazione mediante angioplastica). Dopo un periodo di 6 mesi si è rilevato un significativo miglioramento della sola PA diastolica nel secondo gruppo.

Vi sono però elementi di debolezza del trial. Infatti sono stati esclusi pazienti con RAS che utilizzavano 3 farmaci antipertensivi. Erano pertanto esclusi pazienti con ipertensione resistente e maligna. Inoltre vi era un basso numero di pazienti studiati, un basso utilizzo di stent rispetto alla semplice angioplastica, un'alta percentuale di complicanze. Infine è da rilevare che lo studio risale al 1998.

Lo studio STAR (25) è un trial europeo su 140 pazienti provenienti da 10 Centri con RAS $>50 \%$ e Ccr tra 15 e $80 \mathrm{~mL} /$ min. Tutti i pazienti ricevevano Ace-inibitori e statine. Settantasei pazienti sono stati sottoposti alla sola terapia medica mentre 64 pazienti sono stati trattati con terapia medica più stenting. Dopo un periodo di 2 anni non è stata osservata alcuna differenza tra i 2 gruppi in riferimento alla funzionalità renale, alla pressione arteriosa, alla morbidità cardiovascolare e alla morte.

Lo studio STAR ha anch'esso alcuni punti di debolezza. Le stenosi non erano sempre significative $(>70 \%)$. Nello studio
STAR il $28 \%$ dei pazienti presentavano una stenosi $<50 \%$ che non avrebbe pertanto richiesto l'intervento di rivascolarizzazione. Il 33\% dei pazienti avevano una moderata RAS (50\%-70\%). Inoltre erano inclusi nello studio pazienti con insufficienza renale ed ipertensione solo moderata. Infine più della metà dei pazienti presentavano una patologia unilaterale. Poiché l'endpoint primario era una riduzione della $\mathrm{Ccr}$ $>20 \%$ non è sorprendente che pazienti con patologia unilaterale e stenosi $<70 \%$ non abbiano avuto benefici dalla rivascolarizzazione.

Lo studio ASTRAL (26) ha arruolato 806 pazienti con RAS considerati idonei a trattamento di rivascolarizzazione percutanea. I pazienti presentavano Ccr intorno ai $40 \mathrm{~mL} / \mathrm{min}$ e molti ricevevano ace-inibitori e statine. I pazienti, con criterio randomizzato, sono stati divisi in 2 gruppi. Un gruppo di 403 pazienti è stato sottoposto alla sola terapia medica. Un altro gruppo iniziale di 403 pazienti, poi ridottosi a 335, è stato sottoposto a terapia medica più rivascolarizzazione. Dopo un periodo di studio di 33 mesi non è stata osservata alcuna differenza tra i 2 gruppi in riferimento alla funzionalità renale e pressione arteriosa. Non vi era nessuna differenza neanche nel sottogruppo di pazienti con RAS $>70 \%$.

L'unica differenza significativa era rappresentata dalla diminuita necessità di terapia farmacologica (2.77 stenting vs 2.97 sola terapia medica $\mathrm{p}<0.02$ ).

Vi sono state alcune gravi complicanze, quali 2 decessi e 3 amputazioni, nel gruppo sottoposto a rivascolarizzazione. Anche questo studio presenta alcuni punti di debolezza. Circa il $40 \%$ dei pazienti presentava una stenosi $<70 \%$. Il numero di farmaci antipertensivi prescritti non sempre giustificava l'intervento di rivascolarizzazione secondo le linee guida internazionali. Vi era inoltre notevole variazione sullo stadio d'insufficienza renale. Il $40 \%$ dei pazienti presentavano una creatininemia $<1.7 \mathrm{mg}$, il $25 \%$ avevano normofunzione rena-

TABELLA I - QUATTRO STUDI RANDOMIZZATI E CONTROLLATI SU PAZIENTI CON RAS

\begin{tabular}{|c|c|c|c|c|c|c|}
\hline Trial & $\begin{array}{l}\text { Criteri di selezione } \\
\text { maggiori }\end{array}$ & Pazienti trattati & Controlli & $\begin{array}{l}\text { Outcome } \\
\text { primario }\end{array}$ & Risultati & $\begin{array}{l}\text { Complicanze nei } p z \\
\text { trattati }(\%)\end{array}$ \\
\hline $\begin{array}{l}\text { EMMA } \\
(1998)\end{array}$ & $\begin{array}{l}\text { Stenosi } \geq 60 \% \\
\text { GFR }>50 \mathrm{~mL} / \mathrm{min} \\
\text { PA diastolica }>95 \mathrm{mmHg}\end{array}$ & $\begin{array}{l}\text { PTA con o senza } \\
\text { stent } \\
(23)\end{array}$ & $\begin{array}{l}\text { Terapia } \\
\text { farmacologica } \\
(26)\end{array}$ & Riduzione PA & $\begin{array}{l}\text { Riduzione significativa } \\
\text { della sola PA diastolica }\end{array}$ & $\begin{array}{l}\text { Ematomi (26) } \\
\text { Lesioni renali acute ( } 4.3)\end{array}$ \\
\hline $\begin{array}{l}\text { STAR } \\
(2009)\end{array}$ & $\begin{array}{l}\text { Stenosi } \geq 50 \% \\
\text { GFR } 15-80 \mathrm{~mL} / \mathrm{min} \\
\text { PA stabile }\end{array}$ & $\begin{array}{l}\text { PTA + Stent } \\
(64)\end{array}$ & $\begin{array}{l}\text { Terapia } \\
\text { farmacologica } \\
(76)\end{array}$ & $\begin{array}{l}\text { Riduzione GFR } \\
\geq 20 \%\end{array}$ & $\begin{array}{l}\text { Nessuna differenza } \\
\text { significativa } \\
\text { nell'outcome primario } \\
\text { o nella PA }\end{array}$ & $\begin{array}{l}\text { Morte }(3.2) \\
\text { Ematomi (17) } \\
\text { Lesioni renali acute }(8)\end{array}$ \\
\hline $\begin{array}{l}\text { ASTRAL } \\
(2009)\end{array}$ & $\begin{array}{l}\text { Stenosi senza chiara } \\
\text { indicazione alla } \\
\text { rivascolarizzazione }\end{array}$ & $\begin{array}{l}\text { PTA con o senza } \\
\text { stent } \\
(403)\end{array}$ & $\begin{array}{l}\text { Terapia } \\
\text { farmacologica } \\
(403)\end{array}$ & $\begin{array}{l}\text { Variazione del } \\
\text { reciproco della } \\
\text { creatinina }\end{array}$ & $\begin{array}{l}\text { Nessuna differenza } \\
\text { significativa } \\
\text { nell'outcome } \\
\text { primario, PA ed eventi } \\
\text { cardiovascolari }\end{array}$ & $\begin{array}{l}\text { Morte }(0.7) \\
\text { Ospedalizzazioni } \\
\text { per ematomi }(1.4) \\
\text { Lesioni renali acute }(2.1)\end{array}$ \\
\hline $\begin{array}{l}\text { CORAL } \\
(2006)\end{array}$ & $\begin{array}{l}\text { Stenosi } \geq 60 \% \\
\text { Pressione sistolica } \geq 155 \\
\text { mmHg con } \geq 2 \text { farmaci } \\
\text { antiipertensivi }\end{array}$ & PTA + Stent & $\begin{array}{l}\text { Terapia } \\
\text { farmacologica }\end{array}$ & $\begin{array}{l}\text { Raddoppio della } \\
\text { creatininemia o } \\
\text { necessità di terapia } \\
\text { sostitutiva }\end{array}$ & In corso & \\
\hline
\end{tabular}

EMMA, Essay Multicentrique Medicaments vs Angioplastic; STAR, Stenosis of the Renal Artery; ASTRAL, Angioplasty and Stenting for Renal Artery Lesions; CORAL, Cardiovascular Outcomes in Renal Artherosclerotic Lesions; GRF, glomerular filtration rate; PTA, percutaneous transluminal angioplasty; PA, pressione arteriosa. 
TABELLA II - TERAPIA MEDICA NEI PAZIENTI CON RAS ATEROSCLEROTICO (STUDIO CORAL)

\begin{tabular}{|c|c|c|}
\hline Fattori di rischio & Obiettivi terapeutici & Terapia \\
\hline \multirow[t]{2}{*}{ Ipertensione } & $\mathrm{PA}<140 / 90 \mathrm{mmHg}$ & $\begin{array}{c}\text { ACE inibitori o } \\
\text { sartanici }\left(1^{\circ} \text { scelta }\right)\end{array}$ \\
\hline & $\mathrm{A}<130 / 80 \mathrm{mmHg}$ (diabetici) & $\begin{array}{c}\text { Diuret, } \beta \text {-blocc o } \\
\text { Ca-antag }\left(2^{\circ} \text { scelta }\right)\end{array}$ \\
\hline Dislipidemia & $\mathrm{LDL}<100 \mathrm{mg} / \mathrm{dL}$ & $\begin{array}{c}\text { Inibitori HMG-CoA } \\
\text { redattasi }\end{array}$ \\
\hline Diabete mellito & $\mathrm{HbA} 1 \mathrm{C}<7 \mathrm{mg} / \mathrm{dL}$ & $\begin{array}{c}\text { Insulina, antidiabetici } \\
\text { orali }\end{array}$ \\
\hline Abuso di nicotina & Cessazione del fumo & $\begin{array}{c}\text { Programma di } \\
\text { cessazione del fumo }\end{array}$ \\
\hline Eventi cardiovascolari & $\begin{array}{c}\text { Terapia } \\
\text { antiaggregante }\end{array}$ & $\begin{array}{l}\text { Aspirina, } \\
\text { Clopidogrel }\end{array}$ \\
\hline
\end{tabular}

TABELLA III - INDICAZIONI DELL'AHA PER LA RIVASCOLARIZZAZIONE NELLA STENOSI DELL'ARTERIA RENALE

\footnotetext{
Ipertensione:

Accelerata - improvviso peggioramento della PA prima ben controllata

Refrattaria - resistente ad almeno 3 farmaci antiipertensivi

Maligna - responsabile di danno d'organo

Associata a rene unilateralmente piccolo

Associata ad intolleranza ai farmaci

Alterazioni cardiache associate:

Ricorrenti episodi di edema polmonare non proporzionati al deficit cardiaco

Angina instabile

Improvviso peggioramento della funzione renale:

dopo l'instaurarsi di terapia antiipertensiva in particolare ACE inibitori

e sartanici non attribuibile ad altre cause
}

le. Infine molti avevano patologia solo unilaterale.

Lo studio CORAL (27), di cui è stato finora pubblicato solo il "Trial Design", è uno studio randomizzato controllato che è ancora in corso in USA. Sono stati arruolati 1,080 pazienti con ipertensione resistente o GFR $<60 \mathrm{~mL} / \mathrm{min}$. L'arruolamento è stato ultimato nel Gennaio 2010 e i risultati saranno disponibili nei prossimi anni. Lo studio CORAL, a differenza dei precedenti trial e sulla base delle linee guida internazionali, prevede una rigorosa terapia antipertensiva, la cessazione dal fumo e il trattamento della dislipidemia (Tab. II). Si può anticipare che i risultati del CORAL Study potranno chiarire compiutamente quale opzione terapeutica sia preferibile in quanto il rigore dei criteri di inclusione eviteranno le debolezze dei trial precedenti.

\section{Conclusioni}

Lo stenting dell'arteria renale nel trattamento della RAS è un intervento semplice e gravato da sempre meno effetti collaterali ma non vi è ancora un trial randomizzato e controllato che ne dimostri un significativo beneficio clinico rispetto alla sola terapia farmacologica.

Pertanto la procedura di rivascolarizzazione effettuata in maniera indiscriminata non è più da considerare accettabile. È preferibile un approccio integrato che porti ad una personalizzazione del trattamento. Le linee guida dell'American Heart Association (28), come riportato nella Tabella III, possono essere comunque di aiuto nell'indicazione alla rivascolarizzazione dell'arteria renale.

\section{Riassunto}

La stenosi dell'arteria renale (RAS) è nel $90 \%$ dei casi su base aterosclerotica; in quasi il $10 \%$ dei casi è invece correlata a displasia fibromuscolare. La RAS può essere causa di ipertensione maligna nonché di riduzione della funzionalità renale. È pertanto importante mettere in atto una terapia ottimale per ridurre le complicanze. Vi è un generale consenso sull'efficacia di una rigorosa terapia medica. È invece dibattuta l'utilità di un concomitante intervento di rivascolarizzazione percutanea per migliorare la PA e il GFR. Abbiamo pertanto effettuato una ricerca della letteratura utilizzando PubMed e ricercando i termini "renal artery stenosis, atherosclerotic renal artery stenosis, renovascular hypertension". In questa review analizziamo l'epidemiologia, la fisiopatologia, la diagnosi e, in modo più approfondito, le diverse opzioni terapeutiche alla luce dei più recenti trial clinici.

Parole chiave: Stenosi dell'arteria renale, Stenosi aterosclerotica dell'arteria renale, Ipertensione renovascolare, Rivascolarizzazione percutanea

Dichiarazione di conflitto di interessi: Gli Autori dichiarano di non avere conflitto di interessi.

\section{Indirizzo degli autori:}

Dr.ssa Maria Capuano

Unità Operativa di Nefrologia e Dialisi

Ospedale dei Pellegrini

Via Portamedina alla Pignasecca 41

80134 Napoli

maria.capuano05@libero.it 


\section{Bibliografia}

1. Safian, RD, Textor, SC. Renal-artery stenosis. N Engl J Med 2001; 344: 431-42.

2. Hansen KJ, Edwards MS, Crafen TE, et al. Prevalence of renovascular disease in the elderly: a population-based study. J Vasc Surg 2002; 36: 443-51.

3. Nakashima H, Suzuki $\mathrm{H}$, et al. Angiotensin II regulates vascular and endothelium disfunction. Curr Vasc Pharmacol 2006; 4: 67-78.

4. Baumgartner I, Behrendt P, Rohner P, Baumgartner RW. A validation study on the intraobserver and interobserver reproducibility of renal artery duplex ultrasound. Ultrasound Med Biol 1999; 25: 225-31.

5. Radermacher J, Chavan A, Schaffer J, et al. Detection of significant renal artery stenosis with color Doppler sonography: combining extrarenal and intrarenal approaches to minimize technical failure. Clin Nephrol 2000; 53: 333-43.

6. Soares GM, Murphy TP, Singha MS, Parada A, Jaff M. Renal artery duplex ultrasonography as a screening and surveillance tool to detect renal artery stenosis: a comparison with current reference standard imaging. J Ultrasound Med 2006; 25: 293-8.

7. Vasbinder GB, Nelemans PJ, Kessels AG, Kroon AA, de Leeuw PW, van Engelshoven JM. Diagnostic tests for renal artery stenosis in patients suspected of having renovascular hypertension: a meta-analysis. Ann Intern Med 2001; 135: 401-11.

8. Eklof H, Ahlstrom H, Magnusson A, et al. A prospective comparison of duplex ultrasonography, captopril renography, MRA, and CTA in assessing renal artery stenosis. Acta Radiol 2006; 47: 764-74.

9. Leiner T, de Haan MW, Nelemans PJ, van Engelshoven JM, Vasbinder, GB. Contemporary imaging techniques for the diagnosis of renal artery stenosis. Eur Radiol 2005; 15: 2219-29.

10. Ergun I, Keven K, Uruc I, et al. The safety of gadolinium in patients with stage 3 and 4 renal failure. Nephrol Dial Transplant 2006; 21: 697-700.

11. Chrysochou C, Mendichovszkyl A, Buckley DL, et al. BOLD imaging: a potential predictive biomarker of renal functional outcome following revascularization in atheromatous renovascular disease. Nephrol Dial Transplant 2012; 27(3): 1013-9.

12. Tanemoto M, Suzuki T, Abe M, et al. Hemodynamic index of atheromatous renal artery stenosis for angioplasty. Clin J Am Soc Nephrol 2009; 4: 651-5.

13. Van de Ven PJG, Kaatee R, Beutler JJ, et al. Arterial stenting and balloon angioplasty in ostial atherosclerotic renovascular disease: a randomised trial. Lancet 1999; 353: 282-6.

14. Webster J, Marshall F, Abdalla M, et al. Randomised compari- son of percutaneous angioplasty vs continued medical therapy for hypertensive patients with atheromatous renal artery stenosis. J Hum Hypertens 1998; 12: 329-35.

15. Van Jaarsveld BC, Krijnen P, Pieterman H, et al. The effect of balloon angioplasty on hypertension in atherosclerotic renalartery stenosis. N Engl J Med 2000; 342: 1007-14.

16. Yusuf S. Two decades of progress in preventing vascular disease. Lancet 2002; 360: 2-3.

17. Cianci R, Martina P, Borghesi F, et al. Revascularization versus medical therapy for renal artery stenosis: antihypertensive drugs and renal outcome. Angiology 2011; 62(1): 92-9.

18. Chrysochou C, Foley RN, Young JF, et al. Dispelling the mith: the use of renin angiotensin blockade in atheromatous renovascular disease. Nephrol Dial Transplant 2012; 27(4): 1604-9.

19. Carr TM, Sabri SS, Turba UC, et al Stenting for atherosclerotic renal artery stenosis. Tech Vasc Interv Radiol 2010; 13(2): 134-45.

20. Arthurs Z, Starnes B, Cuadrado D, Sohn V, Cushner H, Andersen $C$. Renal artery stenting slows the rate of renal function decline. J Vasc Surg 2007; 45(4): 726-31.

21. Rabbia C, Pini R. Evidence-based medicine in renal artery stenting. J Cardiovasc Surg 2010; 51(5): 755-63.

22. Steichen O, Amar L, Plouin PF. Primary stenting for atherosclerotic renal artery stenosis. J Vasc Surg 2010; 51(6): 1574-80.

23. Jaff MR, Bates M, Sullivan T, et al. Significant reduction in systolic blood pressure following renal artery stenting in patients with uncontrolled hypertension: results from the HERCULES study. Catheter Cardiovasc Interv 2012; 80: 343-50.

24. Plouin PF, Chatellier G, Darne B, et al. Essay Multicentrique Medicaments vs Angioplastic (EMMA) Study Group. Blood pressure outcome of angioplastic in atherosclerotic renal artery stenosis: a randomized trial. Hypertension 1998; 31: 823-9.

25. Bax L, Woittiez AJ, Kouwenberg HJ, et al. Stent placement in patients with atherosclerotic renal artery stenosis and impaired renal function: a randomized trial. Ann Intern Med 2009; 150: $840-8$.

26. Wheatley K, Ives N, Gray R, et al. Revascularization versus medical therapy for renal-artery stenosis. N Engl J Med 2009; 361: 1953-62.

27. Cooper CJ, Murphy TP, Matsumoto A, et al. Stent revascularization for the prevention of cardiovascular and renal events among patients with renal artery stenosis and systolic hypertension: rationale and design of the CORAL trial. Am Heart J 2006; 152: 59-66.

28. Rundback, JH, Sacks, D, Kent, KC, et al. Guidelines for the reporting of renal artery revascularization in clinical trials. American Heart Association. Circulation 2002; 106: 1572-85. 